\title{
Prognostic value of systemic inflammatory factors NLR, LMR, PLR and LDH in penile cancer
}

\author{
Chen Hu, Yu Bai,, Jun Li, Guoyin Zhang, Libo Yang, Chengwei Bi, Bin Zhao, Yong Yang, Ruiqian Li, Hongyi Wu, \\ Qilin Wang and Yang Qin
}

\begin{abstract}
Background: Penile squamous cell carcinoma (PSCC) represents an important public health problem for developing countries. The major prognostic factors in PSCC are pathological subtype, perineural invasion, lymphovascular invasion, depth of invasion and grade, which are hard to obtain precisely before the operation. Besides, micro-metastases will be detected in about $30 \%$ of intermediate-risk patients with clinically non-palpable inguinal lymph nodes after inguinal lymph node dissection (ILND). It means approximately $70 \%$ of patients are unable to benefit from ILND who might suffered from the complications of surgery. We hope some biomarkers could be found which are able to predict the outcome before surgery and reflect the inguinal lymph nodes metastasis.
\end{abstract}

Methods: A total of 349 consecutive patients of penile cancer in Yunnan cancer hospital in China between October 2002 and December2017. Two hundred twenty-five was succeed to follow-up. The association between NLR, LMR, PLR, LDH and Overall survival (OS), progression free survival (PFS), inguinal lymph node (N stage) was analyzed with K-M analysis, univariable, multivariable logistic regression and Kendall's tau-b correlation coefficient.

Results: Multivariable analysis reveal that only PLR was significant independent factor which is associated with inferior OS and PFS; Age and LDH was associated with inferior OS; Lymph node and metastatic status remained significant for OS and PFS as NCCN and EAU Guidelines indicated; the tumor type, initial treatment and NLR LMR were not significant in predicting both OS and PFS. NLR, LMR and PLR were corresponded to N stage, while LDH was not associated with the $N$ stage based on logistic regression model analysis. NLR, LMR and PLR were found weakly related to N stage through an application of Kendall's tau-b correlation coefficient.

Conclusions: PLR was significant independent factors for OS and PFS, Age and LDH was significant independent factors for OS. NLR, LMR, PLR was corresponded to N stage.

Keywords: Penile cancer, NLR, LMR, PLR, LDH

\section{Background}

Penile squamous cell carcinoma has a low incidence among all cancers, according to the report there are 26, 000 new cases occurring worldwide annually [1]. However, in developing countries such as Africa, Asia, South

\footnotetext{
*Correspondence: 2447519483@qq.com

Department of Urology, Yunnan Cancer Hospital, The Third Affiliated Hospital of Kunming Medical University, Kunming 650118, Yunnan, China
}

America, etc. penile cancer is still a difficult problem, where its incidence varies from 3 to 8.3 cases per 100, 000 [2], especially in southwestern of China. The management of the regional lymph nodes is extremely important for long-term survival of the patient according to the National Comprehensive Cancer Network $(\mathrm{NCCN})$ and the European Association of Urology (EAU) clinical practice guidelines. Both guidelines 
subdivide penile cancer into three groups of low risk, intermediate risk and high risk. Low-risk patients with non-palpable inguinal lymph node (cN0) can be managed by surveillance or dynamic sentinel node biopsy (DSNB), however, due to earlier Inguinal Lymph Node Dissection (ILND) surgery has a better prognosis than ILND after local lymph node recurrence, the indications for ILND recommended by the guidelines are do the operation as long as the inguinal lymph node are palpable, whether it is low-risk, moderate or high risk [3]. However intermediate-risk patients with clinically non-palpable inguinal lymph nodes, only about $30 \%$ of them are able to detect micrometastases after ILND. That means the rest of patients approximately 70\% have no benefit from ILND and suffered from the complications of surgery. So it is crucial to selected the patients who are really benefits from ILND before surgery. Because prognosis of PSCC is highly correlated with lymph nodes stage, plenty of articles have select patients through the prognostic role of biomarkers. The major prognostic factors in PSCC are perineural invasion, pathological subtype, depth of invasion, grade and lymphovascular invasion $[4,5]$. These factors are hard to precisely obtain before the operation. Besides some articles used biomarkers such as p53 and squamous cell carcinoma antigen to predict inguinal lymph node positive rate and prognosis of penile cancer, but they were not applied to clinical practice $[6,7]$. So we hope to find some markers that is associated with inguinal lymph nodes metastatic or can predict the outcomes.

As we known that systemic inflammatory factors play an significant role in cancer progression $[8,9]$. The tumor micro-environment regulated by inflammatory cells is clearly related to cancer progression [8]. Systemic inflammatory factors are obviously reflected by changes of peripheral leukocyte, lymphocytes,neutrophils, monocytes and platelets. These factors above mentioned can reflect the tumor micro environment indirectly [8], so we collected the data of peripheral blood parameters including the neutrophil-to-lymphocyte ratio (NLR), lymphocyte-to-monocyte ratio (LMR), platelet-tolymphocyte ratio (PLR) which have been reported to be independent prognostic factors in various of cancers $[8$, 10-14]. Besides, high lactate dehydrogenase (LDH) has also shown that associated with poor OS in urologic cancer [15].

Recently it has been reported that cancer patients with high NLR is associated with worse OS [16] and worse RFS and CSS $[17,18]$. However, there has been no study evaluating the PLR associated with outcomes of penile cancer. In this retrospective study, we investigated the role of NLR, LMR, PLR and LDH before surgery to predicting whether micro-metastases of inguinal lymph node exist and assessed the prognostic value in penile cancer.

\section{Methods}

We reviewed our electronic medical records system to identify men treated for penile SCC at Yunnan cancer hospital in China between October 2002 and December 2017. Experimental procedures were approved by the Human Ethics Committee of Yunnan Cancer Hospital.A total of 349 consecutive patients treated with ILND in PSCC, 225 was succeed to follow-up, Epidemiological and clinical data, including age, preoperative full blood count results NLR, LMR, PLR LDH and TNM clinical staging, tumor pathology, treatment history and oncological outcome (OS, PFS). Survival analysis was performed by the Kaplan-Meier method for univariable analysis and Cox regression method for multivariable analysis. The NLR was calculated by neutrophil-tolymphocyte ratio, LMR was lymphocyte-to-monocyte ratio and PLR was platelet-to lymphocyte ratio which were obtained through peripheral complete blood count and blood biochemistry before the surgery. We calculate the cut-off point of the Ages, NLR, LMR, PLR and LDH according to the area under curve (AUC) given consideration to sensitivity and specificity levels.

The primary end point were overall survival (OS) and progression free survival (PFS) which defined as the time from the dates of pathological diagnosis to death and tumor progression. Univariable analysis (K-M analysis) was performed to compare the higher and lower groups of NLR, LMR, PLR and LDH. Multivariable analysis (COX logistic regression) were used to verify the individual factors respectively. $P$ values less than 0.05 were considered statistical significance.

We also analyse the relation between the NLR, LMR, PLR, LDH and $\mathrm{N}$ stage through the logistic regression, meantime we use method of Kendall's tau-b correlation coefficient to determine the correlation.

\section{Results}

\section{NLR, LMR, PLR, LDH vs OS/PFS}

A total of 225 patients had collected peripheral complete blood count and blood biochemistry before surgery. The mean $( \pm$ SD) ages were $50.6 \pm 13.4$. The detailed data of patients are summarized in Table 1 . We performed AUC curve to determine the Age, NLR, LMR, PLR and LDH cut-off value which were $53,2.94,4.74,133.5$ and 188.5 respectively. The median interquartile range follow-up was 30 (16.0-63.5) months. Among the 225 patients, 171 was survival which the median interquartile range follow-up was 34 (18.0-84.0) months; 150 was disease free which the median interquartile range follow-up was 33 
Table 1 Patients' characteristics

\begin{tabular}{|c|c|c|c|c|c|c|c|c|c|c|c|c|c|}
\hline \multirow[t]{2}{*}{ Variable } & \multirow[b]{2}{*}{$\begin{array}{l}\text { All } n= \\
225\end{array}$} & \multicolumn{2}{|l|}{ NLR } & \multirow[b]{2}{*}{$p$} & \multicolumn{2}{|l|}{ LMR } & \multirow[b]{2}{*}{$p$} & \multicolumn{2}{|l|}{ PLR } & \multirow[b]{2}{*}{$p$} & \multicolumn{2}{|l|}{$\underline{\mathrm{LDH}}$} & \multirow[b]{2}{*}{$p$} \\
\hline & & $\mathrm{NLR} \leq 2.94$ & $N L R>2.94$ & & $\mathrm{LMR} \leq 4.74$ & $L M R>4.74$ & & $\mathrm{PLR} \leq 133.5$ & $P L R>133.5$ & & $\mathrm{LDH} \leq 188.5$ & $\mathrm{LDH}>188.5$ & \\
\hline Age, y & & & & 0.296 & & & 0.169 & & & 0.566 & & & 0.184 \\
\hline$<53$ & 140 & 103 & 37 & & 55 & 85 & & 105 & 35 & & 115 & 25 & \\
\hline$\geq 53$ & 85 & 54 & 31 & & 45 & 40 & & 55 & 30 & & 65 & 20 & \\
\hline Tumor type & & & & 0.026 & & & 0.091 & & & 0.131 & & & 0.943 \\
\hline benign & 44 & 35 & 9 & & 17 & 27 & & 38 & 6 & & 35 & 9 & \\
\hline well & 96 & 69 & 27 & & 39 & 57 & & 62 & 34 & & 79 & 17 & \\
\hline moderate & 74 & 49 & 25 & & 35 & 39 & & 52 & 22 & & 59 & 15 & \\
\hline poor & 8 & 4 & 4 & & 7 & 1 & & 6 & 2 & & 6 & 2 & \\
\hline others & 3 & 0 & 3 & & 2 & 1 & & 2 & 1 & & 2 & 1 & \\
\hline $\begin{array}{l}\text { Anatomic } \\
\text { stage }\end{array}$ & & & & 0.002 & & & 0.063 & & & 0.005 & & & 0.076 \\
\hline 0 & 44 & 33 & 9 & & 17 & 25 & & 36 & 6 & & 33 & 9 & \\
\hline 1 & 67 & 51 & 16 & & 23 & 44 & & 48 & 19 & & 57 & 10 & \\
\hline$\|$ & 39 & 28 & 11 & & 18 & 21 & & 31 & 8 & & 33 & 6 & \\
\hline III & 62 & 41 & 21 & & 31 & 31 & & 39 & 22 & & 50 & 12 & \\
\hline IV & 15 & 4 & 11 & & 11 & 4 & & 6 & 9 & & 8 & 7 & \\
\hline$N$ stage & & & & 0.001 & & & 0.024 & & & 0.009 & & & 0.053 \\
\hline 0 & 148 & 112 & 36 & & 58 & 90 & & 115 & 33 & & 123 & 25 & \\
\hline 1 & 28 & 15 & 13 & & 18 & 10 & & 15 & 13 & & 24 & 4 & \\
\hline 2 & 36 & 26 & 10 & & 15 & 21 & & 24 & 12 & & 27 & 9 & \\
\hline 3 & 13 & 4 & 9 & & 9 & 4 & & 6 & 7 & & 7 & 6 & \\
\hline M & & & & 0.008 & & & 0.051 & & & 0.146 & & & 0.545 \\
\hline 0 & 222 & 157 & 65 & & 97 & 125 & & 159 & 63 & & 179 & 43 & \\
\hline 1 & 3 & 0 & 3 & & 3 & 0 & & 1 & 2 & & 2 & 1 & \\
\hline $\begin{array}{l}\text { Initial } \\
\text { treatment }\end{array}$ & & & & 0.004 & & & 0.094 & & & 0.863 & & & 0.052 \\
\hline surveillance & 23 & 10 & 13 & & 14 & 9 & & 16 & 7 & & 15 & 8 & \\
\hline surgery & 225 & 147 & 55 & & 85 & 116 & & 144 & 59 & & 166 & 36 & \\
\hline Recurrence & & & & 0.01 & & & 0.635 & & & 0.004 & & & 0.235 \\
\hline no & 150 & 113 & 37 & & 65 & 85 & & 116 & 34 & & 124 & 26 & \\
\hline yes & 75 & 44 & 31 & & 35 & 40 & & 44 & 31 & & 57 & 18 & \\
\hline Vital status & & & & 0.000 & & & 0.001 & & & 0 & & & 0.003 \\
\hline Alive & 171 & 131 & 40 & & 65 & 106 & & 134 & 37 & & 145 & 26 & \\
\hline Dead & 54 & 26 & 28 & & 35 & 19 & & 26 & 28 & & 36 & 18 & \\
\hline
\end{tabular}

(18.0-85.25) months; 29 was alive with disease progression, 8 was dead of other causes.

On Kaplan-Meier analysis, NLR > 2.94 was associated with inferior OS (log-rank $p=0.001$ ), and inferior PFS ( $\log$-rank $p=0.004)$ in Figs. 1 and 2; LMR $>4.74$ was associated with inferior OS (log-rank $p=0.001)$, but not associated with PFS (log-rank $p=0.547$ ) in Figs. 3 and 4; PLR $>133.5$ was associated with inferior OS (log-rank $p<0.001)$ and inferior PFS (log-rank $p=0.001)$ in Figs. 5 and 6. $\mathrm{LDH}<188.5$ was associated with inferior OS (logrank $p=0.004$ ), but not associated with PFS (log-rank $p=0.155)$ in Figs. 7 and 8 .

In univariable analyses, NLR $>2.94$ was associated with inferior OS (HR = 2.967; 95\% CI: 1.739-5.064, $p<0.001)$ and inferior PFS $(\mathrm{HR}=1.944$; 95\% CI: $1.226-3.081, p=$ $0.005)$; $\mathrm{LMR}>4.74$ was associated with inferior OS 


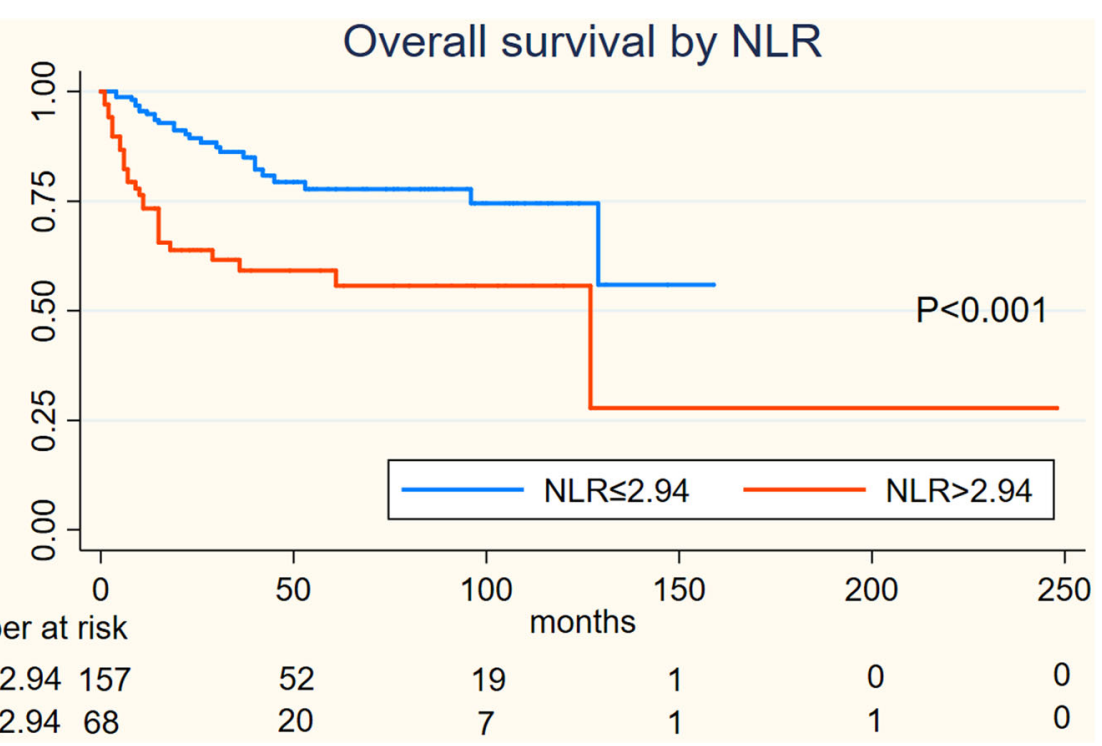

Fig. 1 KaplanMeier curve of NLR for overall survival (OS)

$(\mathrm{HR}=0.394 ;$ 95\% CI: $0.225-0.689, p=0.001)$ and not associated with inferior PFS (HR $=0.871$; 95\% CI: 0.553$1.372, p=0.551)$; PLR $>133.5$ was associated with inferior OS $(\mathrm{HR}=3.439 ; 95 \% \mathrm{CI}: 2.011-5.881, p<0.001)$ and inferior PFS $(\mathrm{HR}=2.194 ; 95 \%$ CI: $1.384-3.478, p=$ $0.001)$; LDH $>188.5$ was associated with inferior OS $(\mathrm{HR}=2.252 ; 95 \% \mathrm{CI}: 1.267-4.002, p=0.006)$ and not associated with inferior PFS $(\mathrm{HR}=1.462 ; 95 \% \mathrm{CI}$ : $0.859-2.487, p=0.162$ ).
We performed a multivariate analysis which reveal NLR was not significant independent factors for $\operatorname{OS}(p=$ $0.516)$ and $\operatorname{PFS}(p=0.594)$; LMR was not associated with $\mathrm{OS}(p=0.97)$ and PFS; PLR was significant independent factors for both $\operatorname{OS}(p=0.001)$ and $\operatorname{PFS}(p=0.02)$; LDH was significant independent factors for $\operatorname{OS}(P=0.035)$ but not for PFS. At multivariate analysis, lymph node status $(P=0.03)$ and metastasis status remained significant for OS and PFS as NCCN or EAU Guidelines

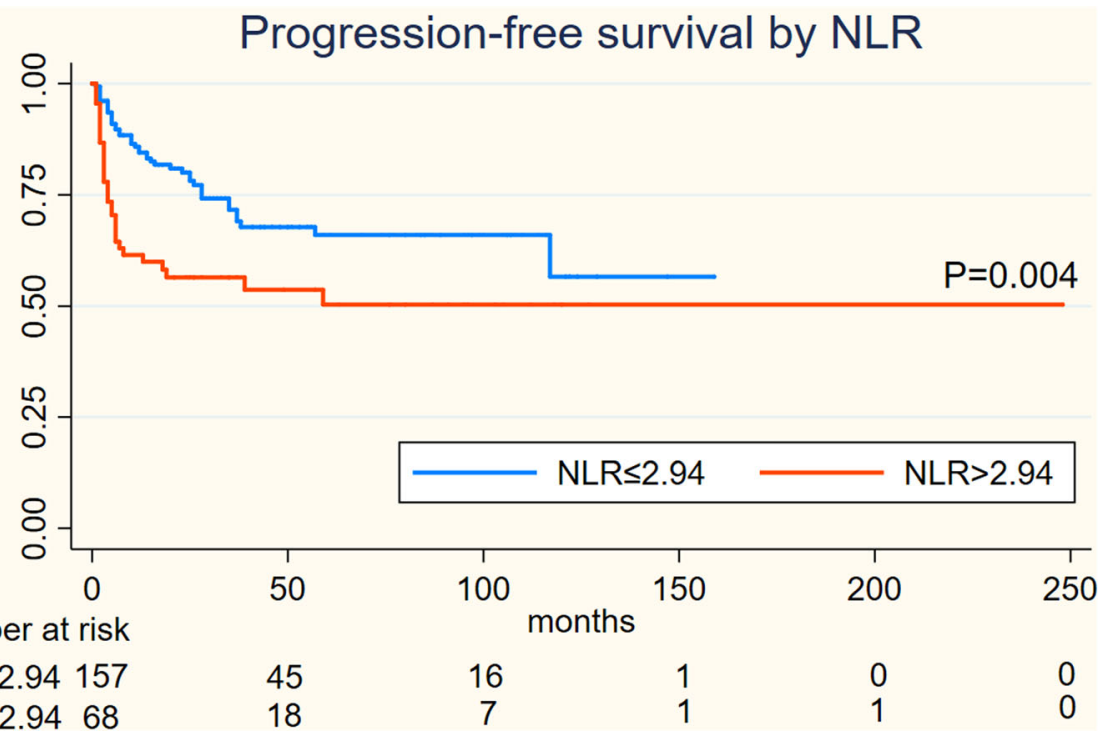

Fig. 2 KaplanMeier curve of NLR for progression free survival (PFS) 


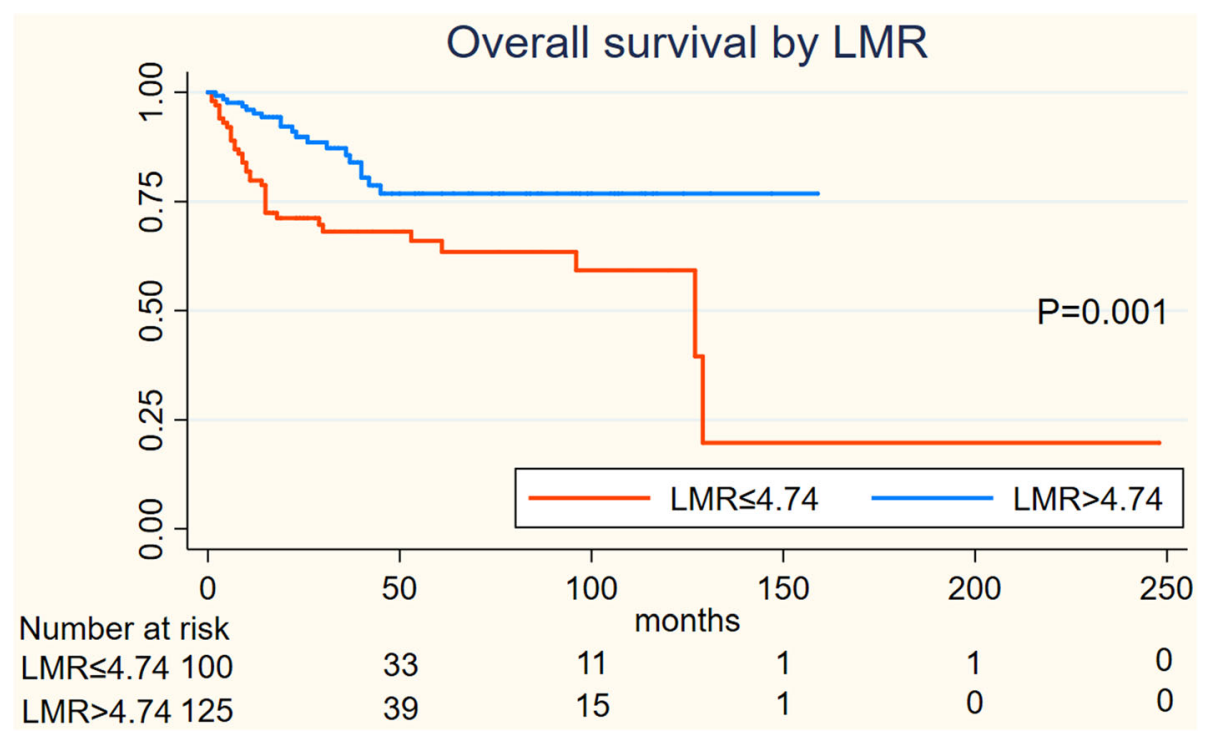

Fig. 3 KaplanMeier curve of LMR for overall survival (OS) and 4

indicated; However, ages, the tumor type and initial treatment was not significant for OS and PFS in multivariable analyses (Table 2).

\section{NLR LMR PLR LDH vs inguinal lymph node-negative/} positive

In logistic regression analyses, NLR $(\mathrm{HR}=2.212 ; 95 \% \mathrm{CI}$ : $1.228-3.985, p=0.008), \quad \mathrm{LMR} \quad(\mathrm{HR}=0.537 ; 95 \% \quad \mathrm{CI}$ : $0.308-0.937, p=0.029)$, PLR (HR $=2.478$; 95\% CI:1.365$4.497, p=0.003)$ was significant corresponded to $\mathrm{N}$ stage which divided into the Node-negative group and Node-positive groups, however the LDH $(p=0.165)$ was not associated with the $\mathrm{N}$ stage. Meantime we also use Kendall's tau-b method to evaluated the correlations, NLR (Kendall's tau-b=0.131, $P=0.017$ ) LMR (Kendall's tau-b $=-0.109, P=0.046$ ) PLR (Kendall's tau-b $=0.161, P=0.003$ ) were weakly correlated to the $\mathrm{N}$ stage. In other words, higher NLR, PLR and lower LMR was more likely to detected pathologically positive inguinal lymph node after ILND. The

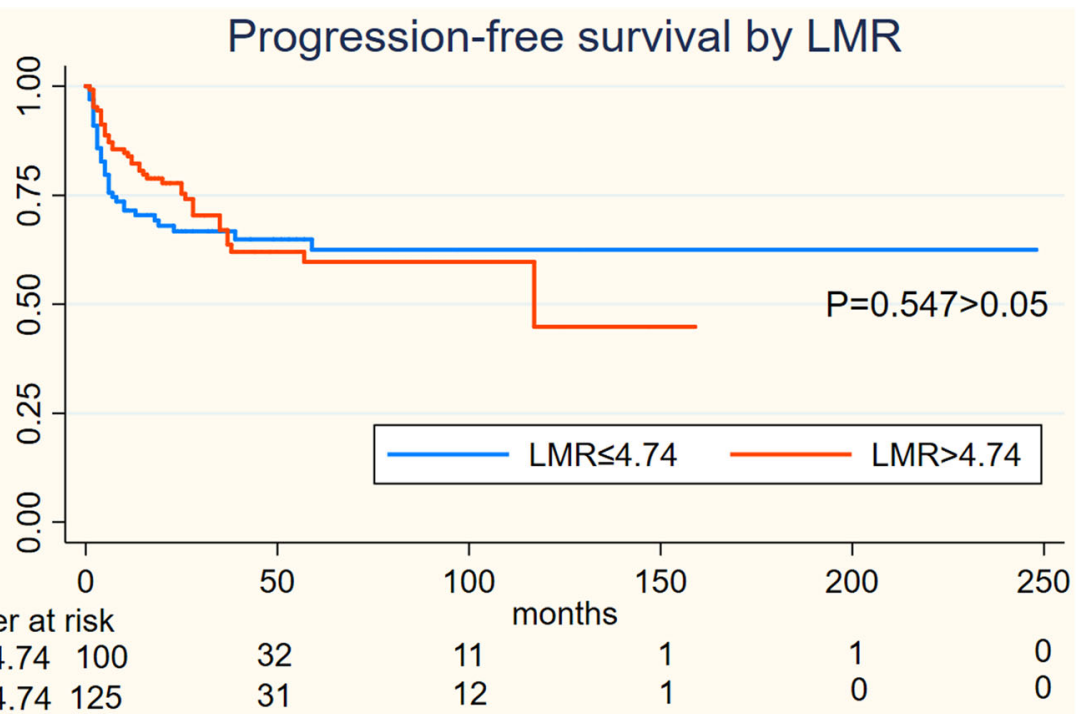

Fig. 4 KaplanMeier curve of LMR for progression free survival (PFS) 


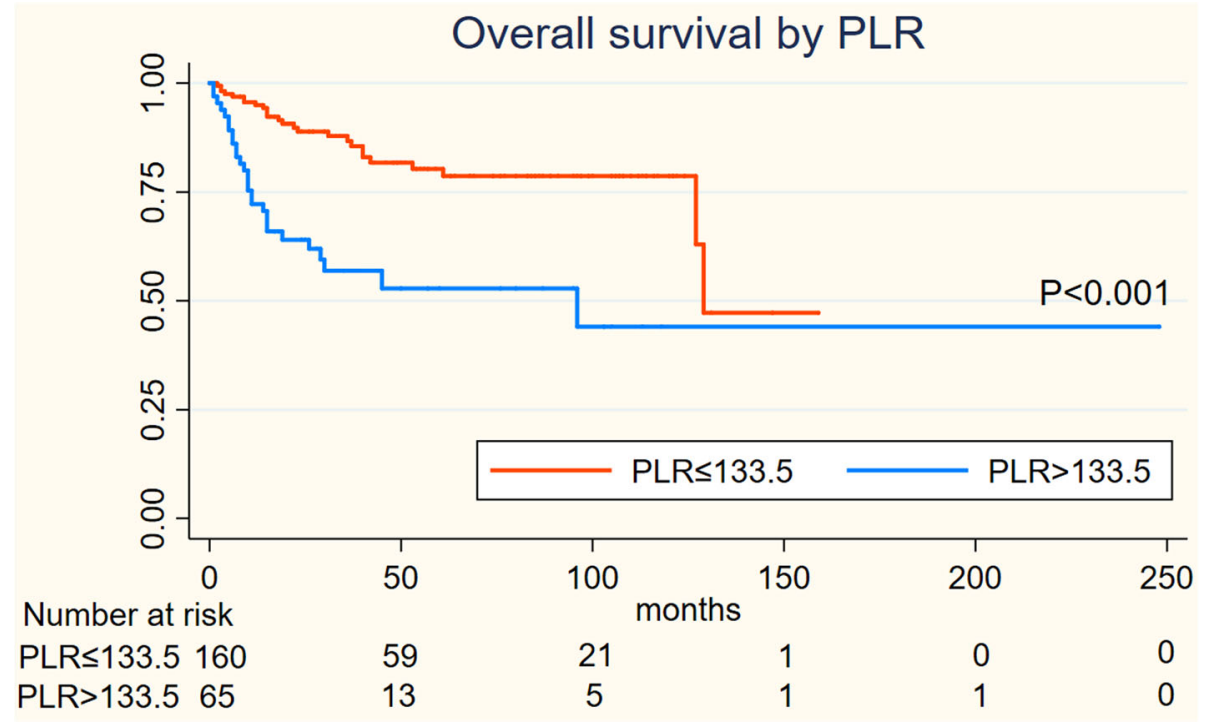

Fig. 5 KaplanMeier curve of PLR for overall survival (OS)

biomarkers of NLR LMR and PLR could predict the pathological outcomes of ILND.

\section{Discussion}

In recent years, the role of inflammatory factors in cancer development and progression has received more attention. The tumor micro-environment may be associated with systemic inflammation. Several types of cancer have been adopt inflammatory factors in prognostic scores for predict the outcome [19] and IMDC scores of advanced renal cell carcinoma also added those factors.

Recently, an elevated ratio of peripheral neutrophils-tolymphocytes (NLR) has been recognized as a poor prognostic indicator in penile cancer, Patients with a high NLR had significantly worse CSS [17, 18] and OS [16];Patients with a low LMR had significantly worse RFS and CSS than those with a high LMR [18].

However, there is no date about the PLR and LDH in penile cancer, this paper aims to collect data from China

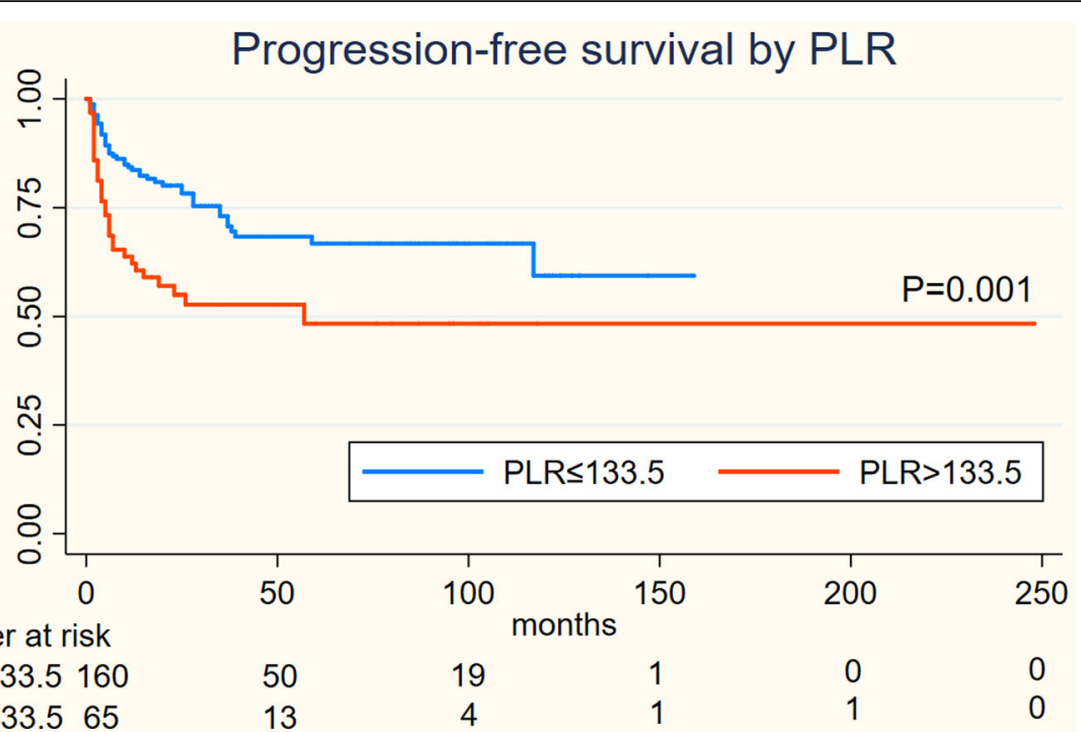

Fig. 6 KaplanMeier curve of PLR for progression free survival (PFS) 


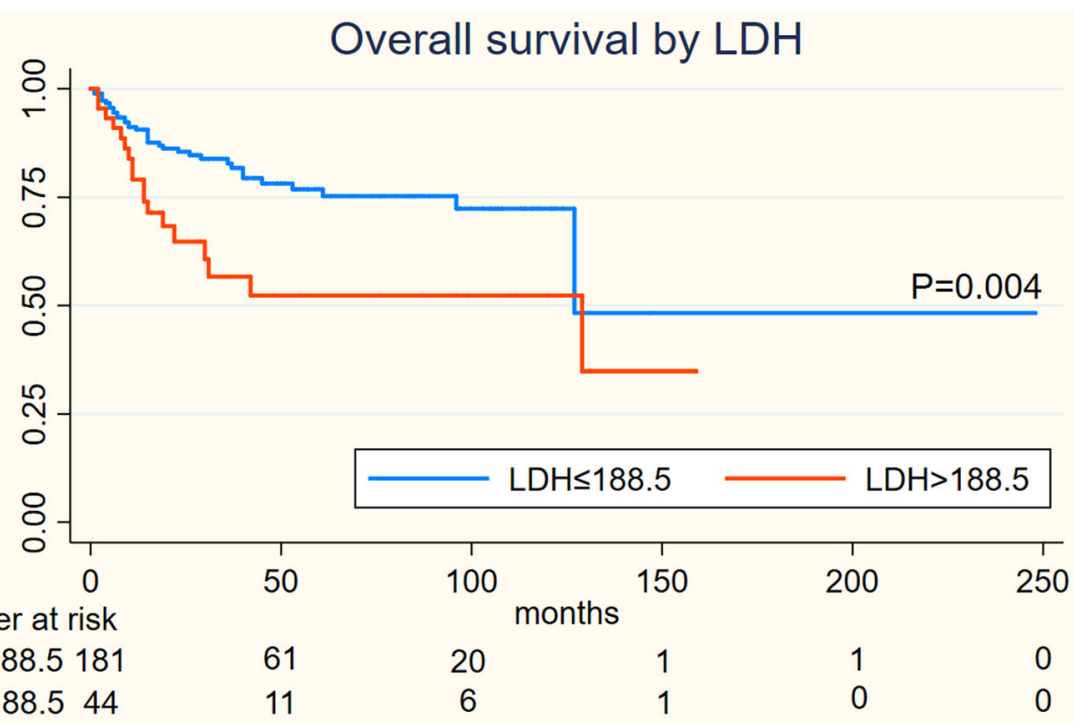

Fig. 7 KaplanMeier curve of LDH for overall survival (OS)

and conduct comparative analysis of NLR, LMR, PLR and $\mathrm{LDH}$, these bio-markers are critical in guiding clinical management decisions and follow-up strategies.

The incidence of penile cancer itself is very low, so it is difficult to analyze with a large sample size. The incidence of penile cancer is relatively high in China, especially in Yunnan Province, so the sample size of this paper is relatively large.

In addition, it is difficult to judge whether there is lymph node metastasis before ILND, therefore, we also hope to assist in judgment whether there is lymph node metastasis from these indicators, so that we can choose the different surgical approach.

We acknowledge the several limitations to this study including inherent biases associated with its retrospective design, insufficient time to follow-up and population heterogeneity. Besides, more than $20 \%$ of the patients were lost to follow-up which may affect the statistical results.

We originally wanted to analyze C-reactive protein (CRP) at the same time. Since the early patients failed to perform CRP testing uniformly, the indicator failed to enter the analysis.

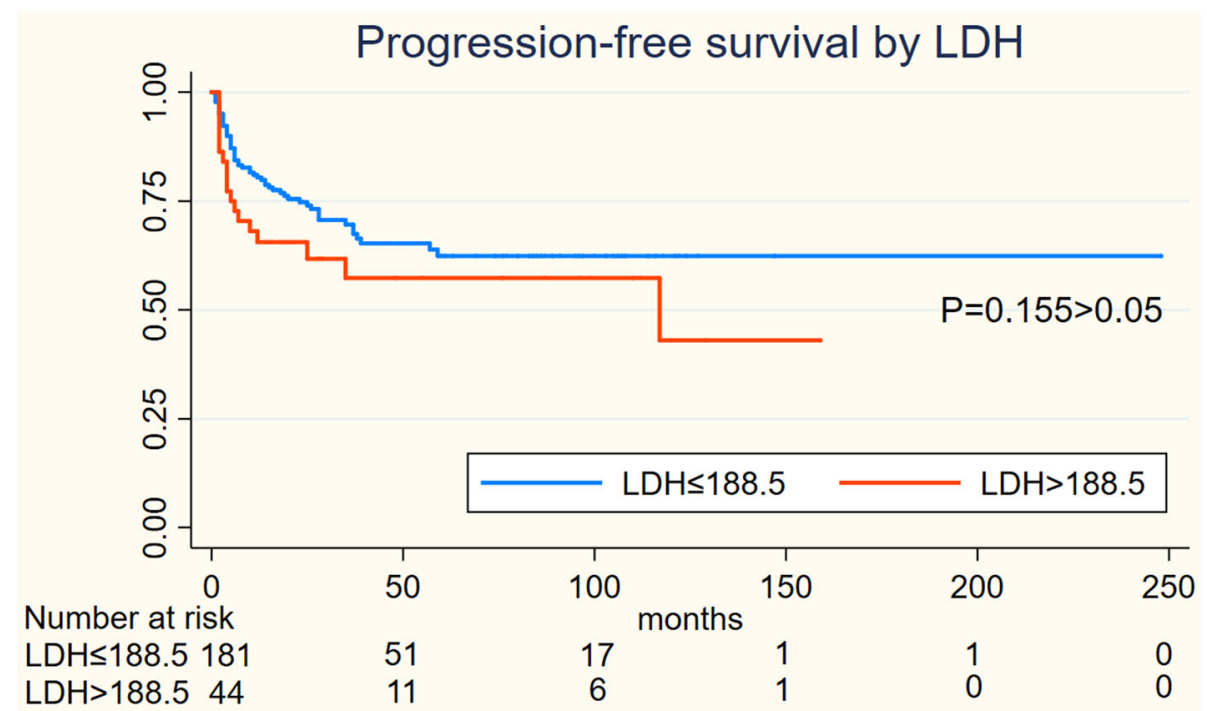

Fig. 8 KaplanMeier curve of LDH for progression free survival (PFS) 
Table 2 Cox regression model for OS and PFS

\begin{tabular}{|c|c|c|c|c|c|c|c|c|c|c|c|c|}
\hline \multirow[t]{3}{*}{ Variables } & \multicolumn{6}{|l|}{ OS } & \multicolumn{6}{|l|}{ PFS } \\
\hline & \multicolumn{3}{|c|}{ Univariable } & \multicolumn{3}{|c|}{ Multivariable } & \multicolumn{3}{|c|}{ Univariable } & \multicolumn{3}{|c|}{ Multivariable } \\
\hline & $\mathrm{HR}$ & $95 \% \mathrm{Cl}$ & $p$ & $\mathrm{HR}$ & $95 \% \mathrm{Cl}$ & $p$ & $\mathrm{HR}$ & $95 \% \mathrm{Cl}$ & $p$ & $\mathrm{HR}$ & $95 \% \mathrm{Cl}$ & $p$ \\
\hline \multicolumn{13}{|l|}{ Ages } \\
\hline$<53$ & reference & $1.077-3.174$ & 0.026 & reference & $1.055-3.983$ & 0.035 & reference & $0.785-1.974$ & 0.352 & & & \\
\hline$\geq 53$ & 1.849 & & & 1.961 & & & 1.245 & & & & & \\
\hline \multicolumn{13}{|l|}{ Tumor type } \\
\hline benign tumor & reference & & 0.002 & reference & & 0.205 & reference & & 0.006 & reference & & 0.135 \\
\hline Well & 0.97 & $0.021-0.448$ & & 0.305 & $0.054-1.715$ & & 0.046 & $0.039-0.970$ & & 0.405 & $0.075-2.186$ & \\
\hline moderate & 0.211 & $0.060-0.737$ & & 0.22 & $0.052-0.938$ & & 0.332 & $0.116-2.070$ & & 0.51 & $0.111-2.336$ & \\
\hline poor & 0.447 & $0.130-1.541$ & & 0.419 & $0.106-1.655$ & & 0.868 & $0.211-3.716$ & & 0.895 & $0.201-3.982$ & \\
\hline others & 0.594 & $0.130-2.719$ & & 0.265 & $0.051-1.378$ & & 0.646 & $0.109-3.944$ & & 0.301 & $0.043-2.112$ & \\
\hline \multicolumn{13}{|c|}{ Inguinal lymph node } \\
\hline negigitve & reference & $5.243-20.918$ & 0.000 & reference & $3.224-15.784$ & 0.000 & reference & $3.096-8.080$ & 0.000 & reference & $2.198-6.250$ & 0.000 \\
\hline positive & 10.472 & & & 7.133 & & & 5.002 & & & 3.706 & & \\
\hline \multicolumn{13}{|l|}{ Metastasis } \\
\hline 0 & reference & $6.637-85.927$ & 0.000 & reference & $2.223-40.238$ & 0.002 & reference & $2.484-26.505$ & 0.001 & reference & 1.184-18.072 & 0.028 \\
\hline 1 & 23.881 & & & 9.458 & & & 8.114 & & & 4.626 & & \\
\hline \multicolumn{13}{|l|}{ Initial treatment } \\
\hline surveillance & reference & $0.222-0.937$ & 0.033 & reference & $0.18-1.006$ & 0.052 & reference & $0.345-1.307$ & 0.241 & & & \\
\hline surgery & 0.456 & & & 0.425 & & & 0.671 & & & & & \\
\hline \multicolumn{13}{|l|}{ NLR } \\
\hline low NLR & reference & $1.739-5.064$ & 0.000 & reference & $0.605-2.718$ & 0.516 & reference & $1.226-3.081$ & 0.005 & reference & $0.679-1.966$ & 0.594 \\
\hline high NLR & 2.967 & & & 1.283 & & & 1.944 & & & 1.115 & & \\
\hline \multicolumn{13}{|l|}{ LMR } \\
\hline low LMR & reference & $0.025-0.689$ & 0.001 & reference & $0.448-2.164$ & 0.97 & reference & $0.553-1.372$ & 0.871 & & & \\
\hline high LMR & 0.394 & & & 0.985 & & & 0.551 & & & & & \\
\hline \multicolumn{13}{|l|}{ PLR } \\
\hline low PLR & reference & $2.011-5.881$ & 0.000 & reference & $1.644-6.171$ & 0.001 & reference & $1.384-3.478$ & 0.001 & reference & $1.104-3.242$ & 0.02 \\
\hline high PLR & 3.439 & & & 3.186 & & & 2.194 & & & 1.892 & & \\
\hline \multicolumn{13}{|l|}{ LDH } \\
\hline low LDH & reference & $1.267-4.002$ & 0.006 & reference & $1.051-3.808$ & 0.035 & reference & $0.859-2.487$ & 0.162 & & & \\
\hline high LDH & 2.252 & & & 2 & & & 1.462 & & & & & \\
\hline
\end{tabular}

\section{Conclusion}

PLR was significant independent factors for OS and PFS, Ages and LDH was significant independent factors for OS. NLR, LMR, PLR was corresponded to $\mathrm{N}$ stage.

\section{Abbreviations}

NLR: Neutrophil-to-lymphocyte ratio; LMR: Lymphocyte-to-monocyte ratio; PLR: Platelet-to-lymphocyte ratio; LDH: Lactate dehydrogenase

Acknowledgments

Not applicable.

\section{Authors' contributions}

YB, CH were participated in the conception and initial design. JL, LBY, CWB performed the clinical treatment of the penile cancer, $\mathrm{CH}, \mathrm{YB}$ was a major contributor in writing the manuscript. $\mathrm{CH}, \mathrm{BZ}, \mathrm{YY}, \mathrm{RQL}$ were participate in articles search, review, data extract, statistical analysis. CH, HYW, GYZ were participate in project development and pictures processing. QLW, YQ were participating in manuscript writing and revising. All authors have read and approved the manuscript.

\section{Funding}

The present study was supported by Applied Basic Research Foundation of Yunnan Province (CN) grant no. 2017FE468(- 215) and 2017FE467(- 191).

Funds were used for purchasing the materials and had no role in the design, collection, analysis, or interpretation of data or writing of this manuscript. 


\section{Availability of data and materials}

The datasets for the current study are available from the corresponding author upon reasonable request.

\section{Ethics approval and consent to participate}

The experimental protocol was approved by the Human Ethics Committee of Yunnan Cancer Hospital. Access and use the data records was granted by The Human Ethics Committee of Yunnan Cancer Hospital. All data was anonymised before its use.

\section{Consent for publication}

Not applicable.

\section{Competing interests}

The authors declare that they have no competing interests.

Received: 6 June 2019 Accepted: 8 May 2020

Published online: 27 May 2020

\section{References}

1. de Martel C, Plummer M, Vignat J, et al. Worldwide burden of cancer attributable to HPV by site, country and HPV type. Int J Cancer. 2017;141(4): 664-70.

2. Wanick FB, Teichner TC, Silva $R$, et al. Squamous cell carcinoma of the penis: clinicopathologic study of 34 cases. An Bras Dermatol. 2011:86(6):1082-91.

3. Kroon BK, Horenblas S, Lont AP, et al. Patients with penile carcinoma benefit from immediate resection of clinically occult lymph node metastases. J Urol. 2005;173(3):816-9.

4. Cubilla AL. The role of pathologic prognostic factors in squamous cell carcinoma of the penis. World J Urol. 2009;27(2):169.

5. Winters BR, Mossanen M, Holt SK, et al. Predictors of nodal upstaging in clinical node negative patients with penile carcinoma: a national cancer database analysis. Urology. 2016;96:29-34.

6. Zhu Y, Zhou XY, Yao XD, et al. The prognostic significance of p53, Ki-67, epithelial cadherin and matrix metalloproteinase-9 in penile squamous cell carcinoma treated with surgery. BJU Int. 2007:100(1):204-8.

7. Zhu Y, Ye DW, Yao XD, et al. The value of squamous cell carcinoma antigen in the prognostic evaluation, treatment monitoring and followup of patients with penile cancer. J Urol. 2008;180(5):2019-23.

8. Coussens LM, ZJN W. Inflammation and cancer. Nature.2002;420(6917):860.

9. Sandler RS, Halabi S, Baron JA, et al. A randomized trial of aspirin to prevent colorectal adenomas in patients with previous colorectal cancer. N Engl J Med. 2003;348(10):883-90.

10. Wang D, Yang JX, Cao DY, et al. Preoperative neutrophil-lymphocyte and platelet-lymphocyte ratios as independent predictors of cervical stromal involvement in surgically treated endometrioid adenocarcinoma. OncoTargets and therapy. 2013:6:211.

11. Wei $X$, Huang F, Wei $Y$, et al. Low lymphocyte-to-monocyte ratio predicts unfavorable prognosis in non-germinal center type diffuse large B-cell lymphoma. Leuk Res. 2014;38(6):694-8.

12. Takeuchi H, Fukuyama S, Kubo N, et al. The prognostic significance of the preoperative platelet-lymphocyte ratio in Japanese patients with localized breast cancer. Adv Breast Cancer Res. 2016:5(02):49.

13. Asher $\mathrm{V}$, Lee J, Innamaa $A$, et al. Preoperative platelet lymphocyte ratio as an independent prognostic marker in ovarian cancer. Clin Transl Oncol. 2011;13(7):499.

14. Kwon HC, Kim SH, Oh SY, et al. Clinical significance of preoperative neutrophil-lymphocyte versus platelet-lymphocyte ratio in patients with operable colorectal cancer. Biomarkers. 2012;17(3):216-22.

15. Zhang $Y, X u T$, Wang $Y$, et al. . Prognostic role of lactate dehydrogenase expression in urologic cancers: a systematic review and meta-analysis. Oncol Res Treat. 2016:39(10):592-604.

16. Azizi M, Peyton CC, Boulware DC, et al. Prognostic value of neutrophil-tolymphocyte ratio in penile squamous cell carcinoma patients undergoing inguinal lymph node dissection. Eur Urol Focus. 2019;5(6):1085-1090.

17. Tan T W, Chia S J, Chong K T. Management of penile cancer in a Singapore tertiary hospital. Arab J Urol. 2017:15(2):123-30.

18. Kasuga J, Kawahara T, Takamoto D, et al. Increased neutrophil-tolymphocyte ratio is associated with disease-specific mortality in patients with penile cancer. BMC Cancer. 2016;16(1):396.
19. McMillan DC. The systemic inflammation-based Glasgow Prognostic Score: a decade of experience in patients with cancer. Cancer Treat Rev. 2013;39(5): 534-40.

\section{Publisher's Note}

Springer Nature remains neutral with regard to jurisdictional claims in published maps and institutional affiliations.
Ready to submit your research? Choose BMC and benefit from:

- fast, convenient online submission

- thorough peer review by experienced researchers in your field

- rapid publication on acceptance

- support for research data, including large and complex data types

- gold Open Access which fosters wider collaboration and increased citations

- maximum visibility for your research: over $100 \mathrm{M}$ website views per year

At $\mathrm{BMC}$, research is always in progress.

Learn more biomedcentral.com/submissions 